\title{
Bit Error Rate Analysis for MC-CDMA Systems in Nakagami-m Fading Channels
}

\author{
Zexian Li \\ Centre for Wireless Communications (CWC), University of Oulu, 90014 Oulu, Finland \\ Email: zexian.li@ee.oulu.fi \\ Matti Latva-aho \\ Centre for Wireless Communications (CWC), University of Oulu, 90014 Oulu, Finland \\ Email:matti.latva-aho@ee.oulu.fi
}

Received 24 February 2003; Revised 22 September 2003

\begin{abstract}
Multicarrier code division multiple access (MC-CDMA) is a promising technique that combines orthogonal frequency division multiplexing (OFDM) with CDMA. In this paper, based on an alternative expression for the $Q$-function, characteristic function and Gaussian approximation, we present a new practical technique for determining the bit error rate (BER) of multiuser MCCDMA systems in frequency-selective Nakagami- $m$ fading channels. The results are applicable to systems employing coherent demodulation with maximal ratio combining (MRC) or equal gain combining (EGC). The analysis assumes that different subcarriers experience independent fading channels, which are not necessarily identically distributed. The final average BER is expressed in the form of a single finite range integral and an integrand composed of tabulated functions which can be easily computed numerically. The accuracy of the proposed approach is demonstrated with computer simulations.
\end{abstract}

Keywords and phrases: multicarrier CDMA, bit error rate, Nakagami fading channel, spread-spectrum communications.

\section{INTRODUCTION}

Multicarrier code division multiple access (MC-CDMA), which efficiently combines CDMA with orthogonal frequency division multiplexing (OFDM), has gained considerable attention as a promising multiple access technique for future mobile communications $[1,2,3,4,5,6,7,8]$. MCCDMA is a spread spectrum technique where the signal is spread in the frequency domain. Since the MC-CDMA technique possesses the advantages of both OFDM and CDMA, it has the properties desirable for future systems such as insensitivity to frequency-selective fading channels, frequency diversity, and the capability of supporting multirate service by applying either multicode or variable spreading factor techniques [1].

Many papers have been dedicated to the bit error rate (BER) analysis of MC-CDMA $[3,4,5,6,7]$. The performance of MC-CDMA has been studied both for the uplink and the downlink of a mobile communication system [3] in which perfect time synchronization among users was assumed. To get the BER, three approximation methods for the distribution of the sum of independently identically distributed (i.i.d.) Rayleigh random variables (r.v.'s) were employed in the paper: the law of large numbers (LLN) approximation, the small parameter approximation and the central limit theorem (CLT) approximation. The authors of [5] analyzed the BER performance of MC-CDMA systems with a frequency offset. The CLT approximation was used in the analysis. A performance analysis using the LLN approximation of an MC-CDMA system employing an antenna array at the base station has been presented in [6]. The bit error probability in multipath channels was analyzed in [7] based on the CLT approximation.

It is well known that approximation methods are not always accurate in practice, thus we have to choose the approximation method according to the system parameters and/or operating environment. For a maximal ratio combining (MRC) receiver operating in a Rayleigh fading channel, the distribution of the sum of exponentially distributed r.v.'s is known to have a gamma distribution from which the exact expression for the average probability of error can be obtained. However, for an equal gain combining (EGC) receiver, finding the distribution of the sum of the independent Rayleigh r.v.'s is more problematic. In [9], Beaulieu offered an infinite series representation of this sum. With the help of characteristic functions of the decision variables, the authors of [10] studied the performance of MC-CDMA with an EGC receiver and Rayleigh fading channels. Nakagami fading channels have received considerable attention in the study of various aspects of wireless systems $[11,12]$. The 


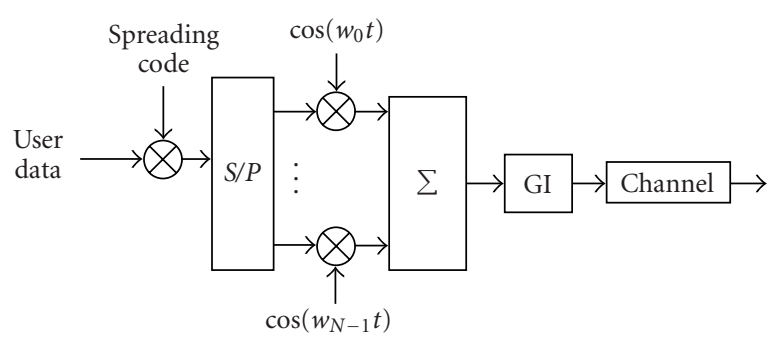

(a)

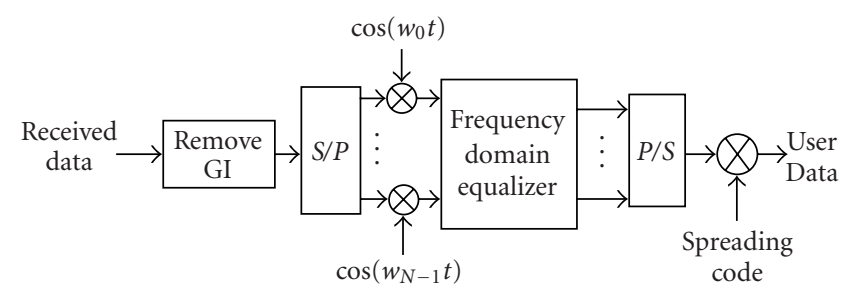

(b)

Figure 1: Block diagram for the MC-CDMA (a) transmitter and (b) receiver (GI: guard interval).

Nakagami distribution provides a more general and versatile way to model wireless channels [13]. The authors investigated the BER performance of MC-CDMA with an EGC receiver and Nakagami- $m$ fading channels [8] with the same fading parameter $m$ on different subcarriers. Although usually the correlation of fading channels amongst subcarriers cannot be ignored, it can be reduced with a properly designed frequency interleaver. Furthermore, the BER performance of MC-CDMA with independent fading channels can provide a helpful benchmark for system design. Motivated by this, the objective of this paper is to present an alternative Gaussian approximation (AGA) approach for deriving the expression for the BER of MC-CDMA with both MRC and EGC in Nakagami- $m$ fading channels where independent fading channels between different subcarriers are assumed. By using an alternative expression for the Gaussian $Q(\cdot)$ function and the characteristic function of Nakagami- $m$ variables, the average BER of an MC-CDMA system can be found.

The rest of this paper is organized as follows. Section 2 gives a description of the MC-CDMA system model. The performance analysis for both MRC and EGC is carried out in Section 3. Section 4 provides a comparison between computer simulation results and analytical results. Finally, Section 5 draws the conclusions.

\section{SYSTEM MODEL}

In this section, the model of an MC-CDMA system is described. We assume that there are $K$ simultaneous users, each having $N$ subcarriers. The block diagrams of the considered MC-CDMA transmitter and receiver with one tap frequency domain equalizers in the uplink are depicted in Figure 1.

\subsection{Transmitter}

Transmitted signal $S_{k}(t)$ corresponding to the block of $M$ data bits of the $k$ th user is

$$
S_{k}(t)=\sqrt{\frac{2 P}{N}} \sum_{m=0}^{M-1} \sum_{n=0}^{N-1} c_{k}[n] b_{k}[m] \cos \left(\omega_{n} t\right) p_{T_{b}}\left(t-m T_{b}\right),
$$

where $P$ is the power of a data bit, $M$ is the packet size, $\left\{c_{k}[n]\right\}$ represents the signature sequence of the $k$ th user, $p_{T_{b}}(t)$ is the rectangular pulse defined over the bit duration $\left[0, T_{b}\right]$, and $b_{k}[m]$ represents the $m$ th input data bit from user $k$ which are independent equiprobable r.v.'s $(\in \pm 1)$ with $E\left[b_{k}(m)\right]=0$ and $E\left[\left|b_{k}(m)\right|^{2}\right]=1 . \omega_{n}$ is the angular frequency of the $n$th subcarrier.

\subsection{Channel model}

Independent, frequency-selective Nakagami- $m$ fading channels for each user are considered. With the proper selection of the number of subcarriers for a user, it is reasonable to assume that each subcarrier undergoes independent frequency-nonselective Nakagami fading. Therefore, the equivalent time-variant complex fading channel for the $k$ th user, $n$th subcarrier can be represented as

$$
H_{k, n}(t ; \tau)=\beta_{k, n}(t) e^{j \theta_{k, n}(t)} \delta\left(\tau-\tau_{k}\right),
$$

where $\tau_{k}$ is the propagation delay for the $k$ th user and $\delta(\cdot)$ is the Dirac delta function. The amplitudes $\left\{\beta_{k, n}(t)\right\}$ are independent Nakagami- $m$ r.v.'s and the phase offsets $\left\{\theta_{k, n}(t)\right\}$ are identical r.v.'s uniformly distributed over $[0,2 \pi)$. The fading amplitude $\beta_{k, n}$ is characterized by a Nakagami- $m$ distribution [13]

$$
p\left(\beta_{k, n}, \Omega_{k, n}\right)=\frac{2 m_{k, n}^{m_{k, n}}}{\Omega_{k, n}^{m_{k, n}}} \frac{\beta_{k, n}^{2 m_{k, n}-1}}{\Gamma\left(m_{k, n}\right)} \exp \left(\frac{-m_{k, n} \beta_{k, n}^{2}}{\Omega_{k, n}}\right)
$$

with the parameters $m_{k, n}=\Omega_{k, n}^{2} / E\left[\left(\beta_{k, n}^{2}-\Omega_{k, n}^{2}\right)^{2}\right] \geq 0.5$ and $\Omega_{k, n}=E\left[\beta_{k, n}^{2}\right], E[\cdot]$ denotes the expectation operator and $\Gamma(\cdot)$ is the Gamma function. The Nakagami assumption on the amplitude implies that $\gamma_{k, n}=\beta_{k, n}^{2}\left(E_{b} / N_{0}\right)\left(E_{b}=P T_{b}\right.$ : the energy per bit) follows the well-known gamma distribution

$$
p\left(\gamma_{k, n}\right)=\frac{m_{k, n}^{m_{k, n}}}{\bar{\gamma}_{k, n}^{m_{k, n}}} \frac{\gamma_{k, n}^{m_{k, n}-1}}{\Gamma\left(m_{k, n}\right)} \exp \left(-\frac{m_{k, n} \gamma_{k, n}}{\bar{\gamma}_{k, n}}\right),
$$

where $\bar{\gamma}_{k, n}=\left(E_{b} / N_{0}\right) \Omega_{k, n}$ is the average signal-to-noise ratio (SNR) per symbol. For the downlink, $H_{k, n}$ is the same for different $k$ at a certain reception point $\{k=0,1, \ldots, K-1\}$.

\subsection{Receiver}

The received signal $r(t)$ can be written as

$$
\begin{aligned}
r(t)= & \sqrt{\frac{2 P}{N}} \sum_{k=0}^{K-1} \sum_{m=0}^{M-1} \sum_{n=0}^{N-1} \beta_{k, n}(t) c_{k}[n] b_{k}[m] \\
& \times \cos \left(\omega_{n} t+\theta_{k, n}(t)\right) p_{T_{b}}\left(t-m T_{b}-\tau_{k}\right)+n(t),
\end{aligned}
$$


where $n(t)$ is the additive white Gaussian noise (AWGN) with a double-sided power spectral density of $N_{0} / 2$.

The insertion of an equalizer in the frequency domain or time domain is necessary to upgrade the performance of the system by multiplying each subcarrier by the factor $G_{k, n}(m)$ in the $m$ th bit interval [14]. Without the loss of generality, we consider the signal from the first user as the desired signal. With coherent demodulation, the decision variable $v_{0}$ of the $m$ th data bit of the first user is given by

$$
v_{0}=\frac{1}{T_{b}} \int_{m T_{b}}^{(m+1) T_{b}} r(t) \sum_{n=0}^{N-1} c_{0}[n] G_{0, n}(m) \cos \left(\omega_{n} t+\theta_{k, n}(t)\right) d t
$$

where it has been assumed that one data bit occupies all subcarriers $^{1}$ and the receiver is synchronized with the desired user $(k=0)$. The channel fading and phase shift variables are assumed to be constant over the time interval $\left[m T_{b},(m+1) T_{b}\right]$ and are denoted by $\beta_{k, n}(m)$ and $\theta_{k, n}(m)$.

In this paper, we have paid attention to the two commonly and effectively used combining methods: MRC $\left(G_{0, n}(m)=\beta_{0, n}(m)\right)$ and $\operatorname{EGC}\left(G_{0, n}(m)=1\right)[14,15]$. For brevity, the time index $m$ is omitted in the following.

\section{PERFORMANCE ANALYSIS}

An alternative representation of the $Q$-function was presented in [16] and leads to a convenient method for performance analysis. By applying the Q-function

$$
Q(x)=\frac{1}{\pi} \int_{0}^{\pi / 2} \exp \left(-\frac{x^{2}}{2 \sin ^{2} \theta}\right) d \theta, \quad x \geq 0
$$

and the characteristic function of Nakagami- $m$ fading r.v.'s, the bit error probability of an MC-CDMA system can be evaluated.

In order to be more general, the uplink direction is considered. For simplicity, it is assumed that different subcarriers experience an i.i.d. fading channel, although identical fading channels are not necessary for the analysis. Assuming that the users are time synchronous, after demodulation and combining subcarrier signals, the decision variable in (6) can be written as

$$
v_{0}=S+I+\eta \text {, }
$$

where $S$ represents the desired signal term, $I$ is the multiple access interference (MAI) from other users, and $\eta$ is the AWGN term.

\subsection{Performance of MRC}

With $G_{0, n}=\beta_{0, n}$ and from (6), (8), we get the desired signal of (8) as

$$
S=\sqrt{\frac{P}{2 N}} \sum_{n=0}^{N-1} b_{0} \beta_{0, n}^{2}
$$

\footnotetext{
${ }^{1}$ Higher data rates can be obtained by using a small spreading factor (SF), that is, subcarriers are used by different data bits. For SF $=1$, the system becomes OFDM.
}

$\eta$ is a Gaussian random variable with zero mean and variance $\sigma_{\eta}^{2}=\left(N_{0} / 4 T_{b}\right) \sum_{n=0}^{N-1} \beta_{0, n}^{2}$. The MAI term $I$ can be expressed as follows:

$$
I=\sqrt{\frac{P}{2 N}} \sum_{k=1}^{K-1} \sum_{n=0}^{N-1} b_{k} c_{k}[n] c_{0}[n] \beta_{k, n} \beta_{0, n} \cos \tilde{\theta}_{k, n}
$$

where $\tilde{\theta}_{k, n}=\theta_{0, n}-\theta_{k, n}$. $\theta_{0, n}$ and $\theta_{k, n}$ are i.i.d. r.v.'s, uniformly distributed over $[0,2 \pi)$. According to [17], the probability density function of $\tilde{\theta}_{k, n}$ can be easily obtained and $E\left[\cos \tilde{\theta}_{k, n}\right]=0$. Since $\beta_{k, n}$ and $\theta_{k, n}(k=1,2, \ldots, K-1 ; n=$ $0,1, \ldots, N-1)$ are i.i.d. r.v.'s, all $(K-1) \times N$ terms in the summation of (10) are uncorrelated with zero means. Assuming that there is no near-far problem, MAI can be approximated by a conditional Gaussian random variable with zero mean and variance

$$
\sigma_{I}^{2}=E\left[I^{2}\right]=\frac{P}{2 N}(K-1) E\left[\beta_{k, n}^{2}\right] E\left[\cos ^{2} \tilde{\theta}_{k, n}\right] \sum_{n=0}^{N-1} \beta_{0, n}^{2},
$$

where $E\left[\cos ^{2} \tilde{\theta}_{k, n}\right]=1 / 2$.

We see that $v_{0}$ is a conditional Gaussian variable conditioned on $\left\{\beta_{0, n}\right\}$. Since $\eta$ and $I$ are mutually independent, the probability of error using BPSK modulation conditioned on $\left\{\beta_{0, n}\right\}$ is simply given by [18]

$$
\operatorname{Pr}\left(\operatorname{error} \mid \beta_{0, n}\right)=Q\left(\sqrt{\frac{S^{2}}{\left(\sigma_{\eta}^{2}+\sigma_{I}^{2}\right)}}\right)
$$

To compute the average BER, we must statistically average (12) over the joint probability density function $p_{\beta}\left(\beta_{0,0}, \ldots, \beta_{0, N-1}\right)$ of the fading amplitudes. Using the alternative $Q$-function (7) and the assumption of independent fading channels at different subcarriers, the average BER can be expressed as

$$
\begin{aligned}
& P_{e}=\int_{0}^{\infty} \cdots \int_{0}^{\infty} \frac{1}{\pi} \int_{0}^{\pi / 2} \exp \left(-\frac{S^{2} /\left(\sigma_{\eta}^{2}+\sigma_{I}^{2}\right)}{2 \sin ^{2} \phi}\right) \\
& \times p_{\beta_{0,0}}\left(\beta_{0,0}\right), \ldots, p_{\beta_{0, N-1}} \\
& \times\left(\beta_{0, N-1}\right) d \beta_{0,0} \cdots d \beta_{0, N-1} d \phi \\
&=\frac{1}{\pi} \int_{0}^{\pi / 2} \prod_{n=0}^{N-1} I_{0, n}\left(\operatorname{SINR}_{0, n}, \phi\right) d \phi,
\end{aligned}
$$

where $\mathrm{SINR}_{0, n}$ is the average signal to interference plus noise ratio (SINR) for the $n$th subcarrier of the first user and the following equation has been used:

$$
\frac{S^{2}}{\sigma_{\eta}^{2}+\sigma_{I}^{2}}=\frac{1}{N / 2+\left(E_{b} / N_{0}\right)((K-1) / 2)} \sum_{n=0}^{N-1} \gamma_{0, n} \text {. }
$$


By using (4) and $\int_{0}^{\infty} x^{v} \exp (-a x) d x=\Gamma(v+1) / a^{v+1}(a>$ $0, v>-1)$ [19], $I_{0, n}$ can be expressed as

$$
\begin{aligned}
I_{0, n}= & \int_{0}^{\infty} \exp \left(-\frac{\gamma_{0, n}}{2 \sin ^{2} \phi\left(N / 2+\left(E_{b} / N_{0}\right)((K-1) / 2)\right)}\right) \\
& \times p\left(\gamma_{0, n}\right) d \gamma_{0, n} \\
= & \left(1+\frac{\operatorname{SINR}_{0, n}}{m_{0, n} \sin ^{2} \phi}\right)^{-m_{0, n}} .
\end{aligned}
$$

The average $\operatorname{SINR}_{0, n}$ for the $n$th subcarrier of the first user can be obtained as

$$
\operatorname{SINR}_{0, n}=\frac{(P / N) E\left[\beta_{0, n}^{2}\right]}{N_{0}+\sum_{k=1}^{K-1}(P / N) E\left[\beta_{k, n}^{2}\right]} .
$$

If all $N$ subcarriers are identically distributed with the same average SINR per bit, then (13) simplifies further to

$$
P_{e}=\frac{1}{\pi} \int_{0}^{\pi / 2}\left[I_{0, n}\left(\operatorname{SINR}_{0, n}, \phi\right)\right]^{N} d \phi .
$$

Since a multiuser system is considered in this paper, the average BER of the system is given by

$$
\mathrm{BER}=\frac{1}{K} \sum_{k=0}^{K-1} P_{e}(k) .
$$

Using (13)-(18), we can obtain the average BER of the MCCDMA system with MRC by using the simple form of a single integral with finite limits and an integrand composed of an elementary function.

\subsection{Performance of EGC}

The EGC equalizer is of importance because the enhancement of MAI due to MRC can be alleviated by EGC. The decision of the $m$ th data bit of the first user is used during the analysis. Similar to MRC, the conditional BER of the system with EGC can be obtained as

$$
\operatorname{Pr}\left(\operatorname{error} \mid \beta_{0, n}\right)=Q\left(\sqrt{\frac{S^{2}}{\left(\sigma_{\eta}^{2}+\sigma_{I}^{2}\right)}}\right),
$$

where the expressions for $S, \eta$, and $I$ are different from those of the MRC receiver and can be derived from (6) and (8).

The desired signal with perfect channel estimation can be expressed as

$$
S=\sqrt{\frac{P}{2 N}} \sum_{n=0}^{N-1} b_{0} \beta_{0, n}
$$

$\eta$ is a Gaussian random variable with zero mean and variance $\sigma_{\eta}^{2}=N N_{0} / 4 T_{b}$. The MAI term $I$ can be written in the form of

$$
I=\sqrt{\frac{P}{2 N}} \sum_{k=1}^{K-1} \sum_{n=0}^{N-1} b_{k} c_{k}[n] c_{0}[n] \beta_{k, n} \cos \tilde{\theta}_{k, n}
$$

where $\tilde{\theta}_{k, n}$ has the same meaning as in (10). The term $I$ can be approximated by a Gaussian random variable with zero mean and variance

$$
\sigma_{I}^{2}=E\left[I^{2}\right]=\frac{P}{2}(K-1) E\left[\beta_{k, n}^{2}\right] E\left[\cos ^{2} \tilde{\theta}_{k, n}\right] .
$$

Using the alternative representation of the $Q$-function (7), the average BER can be expressed as

$$
\begin{aligned}
P_{e}=\int_{0}^{\infty} \cdots \int_{0}^{\infty} \frac{1}{\pi} \int_{0}^{\pi / 2} & \exp \left(-\frac{(P / 2 N) /\left(\sigma_{\eta}^{2}+\sigma_{I}^{2}\right)}{2 \sin ^{2} \phi}\left(\sum_{n=0}^{N-1} \beta_{0, n}\right)^{2}\right) \\
& \times p_{\beta_{0,0}}\left(\beta_{0,0}\right), \ldots, p_{\beta_{0, N-1}}\left(\beta_{0, N-1}\right) \\
& \times d \phi d \beta_{0,0} \cdots d \beta_{0, N-1} .
\end{aligned}
$$

We extended the technique of [20] to an MC-CDMA system with multiple users. By changing variables, (23) becomes

$$
P_{e}=\int_{0}^{\infty} \frac{1}{\pi} \int_{0}^{\pi / 2} \exp \left(-\frac{A^{2}}{2 \sin ^{2} \phi} \lambda^{2}\right) p_{\lambda}(\lambda) d \lambda d \phi,
$$

where

$$
A=\sqrt{\frac{P}{2 N\left(\sigma_{\eta}^{2}+\sigma_{I}^{2}\right)}} \quad \lambda=\sum_{n=0}^{N-1} \beta_{0, n}
$$

and $\lambda$ denotes the sum of the fading amplitudes after combining.

Next, according to the definition of the characteristic function, the term $p_{\lambda}(\lambda)$ could be obtained by employing the characteristic function of the Nakagami- $m$ fading channel

$$
p_{\lambda}(\lambda)=\frac{1}{2 \pi} \int_{-\infty}^{\infty} \psi_{\lambda}(j v) e^{-j v \lambda} d v .
$$

Since the fading experienced by different subcarriers is assumed to be mutually independent, the characteristic function of $\lambda$ simply equals the product of the characteristic function of individual components, leading to

$$
\psi_{\lambda}(j v)=\prod_{n=0}^{N-1} \psi_{\beta_{0, n}}(j v) .
$$

Thus (26) can be of the form

$$
p_{\lambda}(\lambda)=\frac{1}{2 \pi} \int_{-\infty}^{\infty}\left[\prod_{n=0}^{N-1} \psi_{\beta_{0, n}}(j v)\right] e^{-j v \lambda} d v .
$$

By combining (28) and (24), we get

$$
\begin{aligned}
P_{e}= & \frac{1}{2 \pi^{2}} \int_{0}^{\pi / 2} \int_{-\infty}^{\infty}\left[\prod_{n=0}^{N-1} \psi_{\beta_{0, n}}(j v)\right] \\
& \times \underbrace{\int_{0}^{\infty} \exp \left(-\frac{A^{2}}{2 \sin ^{\phi}} \lambda^{2}-j v \lambda\right) d \lambda d v d \phi .}_{J(v, \phi)}
\end{aligned}
$$


The integral of $J(v, \phi)$ can be obtained as [16]

$$
\begin{aligned}
J(v, \phi)= & (X(\phi)+j Y(v, \phi)) \exp \left(-\frac{\sin ^{2} \phi}{2 A^{2}} v^{2}\right) \\
= & \sqrt{X^{2}(\phi)+Y^{2}(v, \phi)} \exp \left[j \arctan \left(\frac{Y(v, \phi)}{X(\phi)}\right)\right] \\
& \times \exp \left(-\frac{\sin ^{2} \phi}{2 A^{2}} v^{2}\right),
\end{aligned}
$$

where ${ }_{1} F_{1}(\cdot ; \cdot ; \cdot)$ is the Kummer confluent hypergeometric function [21] and

$$
\begin{gathered}
X(\phi)=\sqrt{\frac{\pi}{2}} \frac{\sin \phi}{A}, \\
Y(\nu, \phi)=-{ }_{1} F_{1}\left(\frac{1}{2} ; \frac{3}{2} ; \frac{\sin ^{2} \phi}{2 A^{2}} v^{2}\right) \frac{v \sin ^{2} \phi}{A^{2}} .
\end{gathered}
$$

Generally speaking, the characteristic function of a random variable will be a complex quantity and hence the product of the characteristic function in (27) will be also complex. However, since the average BER is real, it is sufficient to consider only the real part of the right side of (29), which yields

$$
P_{e}=\frac{1}{2 \pi^{2}} \int_{0}^{\pi / 2} \int_{-\infty}^{\infty} \mathfrak{R}\left(\left[\prod_{n=0}^{N-1} \psi_{\beta_{0, n}}(j v)\right] J(v, \phi)\right) d v d \phi,
$$

where $\mathfrak{R}(\cdot)$ denotes the real part.

Next we elaborate the expression of the characteristic function corresponding to the Nakagami- $m$ fading channel. By definition, the characteristic function of $\beta_{0, n}$ is given by $\psi_{\beta_{0, n}}(j v)=E\left[\exp \left(j \beta_{0, n} v\right)\right]$. It can be expressed as [20]

$$
\begin{aligned}
\psi_{\lambda}(j v)= & \prod_{n=0}^{N-1} \psi_{\beta_{0, n}}(j v) \\
= & \prod_{n=0}^{N-1}\left[\sqrt{U_{0, n}^{2}(v)+V_{0, n}^{2}(v)} \exp \left(j \arctan \left(\frac{V_{0, n}(v)}{U_{0, n}(v)}\right)\right)\right] \\
& \times \exp \left[-\sum_{n=0}^{N-1} \frac{\Omega_{0, n} v^{2}}{4 m_{0, n}}\right]
\end{aligned}
$$

in which

$$
\begin{gathered}
U_{0, n}={ }_{1} F_{1}\left(\frac{1}{2}-m_{0, n} ; \frac{1}{2} ; \frac{\Omega_{0, n} v^{2}}{4 m_{0, n}}\right), \\
V_{0, n}=\frac{\Gamma\left(m_{0, n}+1 / 2\right)}{\Gamma\left(m_{0, n}\right)} \sqrt{\frac{\Omega_{0, n}}{m_{0, n}}} v_{1} F_{1}\left(1-m_{0, n} ; \frac{3}{2} ; \frac{v^{2} \Omega_{0, n}}{4 m_{0, n}}\right) .
\end{gathered}
$$

Substituting (33) and (30) into (32) gives

$$
\begin{aligned}
P_{e}=\frac{1}{2 \pi^{2}} \int_{0}^{\pi / 2} \int_{-\infty}^{\infty} & \exp \left[-\left(\frac{\sin ^{2} \phi}{2 A^{2}}+\sum_{n=0}^{N-1} \frac{\Omega_{0, n}}{4 m_{0, n}}\right) v^{2}\right] \\
& \times W(v, \phi) d v d \phi,
\end{aligned}
$$

where

$$
\begin{gathered}
W(v, \phi)=R(v, \phi) \cos \Theta(v, \phi), \\
R(v, \phi)=\sqrt{X^{2}(\phi)+Y^{2}(v, \phi)} \prod_{n=0}^{N-1} \sqrt{U_{0, n}^{2}(v)+V_{0, n}^{2}(v)}, \\
\Theta(v, \phi)=\arctan \left(\frac{Y(v, \phi)}{X(\phi)}\right)+\sum_{n=0}^{N-1} \arctan \left(\frac{V_{0, n}(v)}{U_{0, n}(v)}\right) .
\end{gathered}
$$

Finally, letting $\eta(\phi)=\sin ^{2} \phi / 2 A^{2}+\sum_{n=0}^{N-1}\left(\Omega_{0, n} / 4 m_{0, n}\right)$ and changing the variables as $x=\sqrt{\eta(\phi)} v$, the inner infinite integral can be derived as

$$
\int_{-\infty}^{\infty} W\left(\frac{x}{\sqrt{\eta(\phi)}}, \phi\right) \exp \left(-x^{2}\right) d x
$$

which can be readily evaluated by the Gaussian-Hermite quadrature formula [21, 22],

$$
\begin{aligned}
\int_{-\infty}^{\infty} W\left(\frac{x}{\sqrt{\eta(\phi)}}, \phi\right) \exp \left(-x^{2}\right) d x \\
=\sum_{i=1}^{N_{p}} H_{x_{i}} W\left(\frac{x_{i}}{\sqrt{\eta(\phi)}}, \phi\right)+R_{N_{p}}
\end{aligned}
$$

where $N_{p}$ is the order of the Hermite polynomial $H_{N_{p}}(\cdot)$ and $x_{i}$ is the $i$ th zero of the $N_{p}$-order Hermite polynomial. $H_{x_{i}}$ are the weight factors of the $N_{p}$-order Hermite polynomial and are given by

$$
H_{x_{i}}=\frac{2^{N_{p}-1} N_{p} ! \sqrt{\pi}}{N_{p}^{2}\left[H_{N_{p}-1}\left(x_{i}\right)\right]^{2}} .
$$

The remainder of (38) is

$$
R_{N_{p}}=\frac{N_{p} ! \sqrt{\pi}}{2^{n}(2 n) !} W^{\left(2 N_{p}\right)}\left(\frac{\sqrt{2} A}{\sin \phi} \xi, \phi\right) \quad(-\infty<\xi<\infty) .
$$

The order number of $N_{p}$ can be properly selected by taking both complexity and accuracy into consideration. Because of the symmetry of the Hermite polynomials about the origin, the nonzero roots occur in pairs $\pm x_{i}$, and the corresponding weight coefficients obey the symmetry relation $H_{x_{i}}=H_{x_{N_{p}-i}}$. Both the zeros and the weight factors of the $N_{p}$-order Hermite polynomial are tabulated in $[21,22]$ for various polynomial orders $N_{p}$. Thus yielding the final result in the form of a single finite integral on $\phi$, namely,

$$
P_{e}=\frac{1}{2 \pi^{2}} \int_{0}^{\pi / 2} \frac{1}{\sqrt{\eta(\phi)}}\left[\sum_{i=1}^{N_{p}} H_{x_{i}} W\left(\frac{x_{i}}{\sqrt{\eta(\phi)}}, \phi\right)\right] d \phi .
$$

The average BER of a system with multiple users is obtained by averaging (41) over individual users $P_{e}$. 


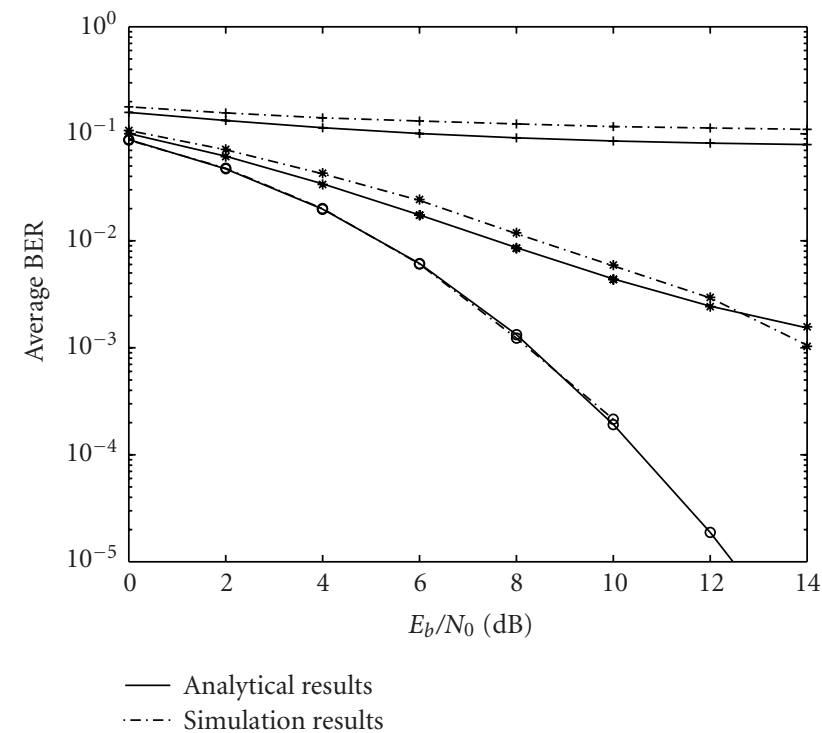

FIgURE 2: BER as a function of $E_{b} / N_{0}(\mathrm{~dB})$ for the MRC receivers of the uplink with different numbers of users in a fading channel. The methods used are AGA analysis and computer simulations $(N=8$; $\circ$ : single user; $*: 2$ active users; $+: 8$ active users).

\section{NUMERICAL AND SIMULATION RESULTS}

In this section, both computer simulations and a theoretical analysis are carried out to investigate the BER performance of an MC-CDMA system with multiple active users in a Nakagami- $m$ fading channel. The fading channels used in computer simulations are Rayleigh (corresponding to $m=1$ ) fading channels and Nakagami- $m$ fading channels $(m=2$ is selected). Both the uplink and downlink are considered here. The simulated system utilizes Walsh-Hadamard (WH) codes as signature sequences. The number of subcarriers is equal to the length of the signature sequence. To calculate the BER, it is assumed that the mean power of each interfering user is equal to the mean power of the desired signal. It is also assumed that the uplink users are synchronous within a cyclic prefix. A flat fading channel on each subcarrier is used and i.i.d. fading among different subcarriers is assumed in this section.

Figure 2 shows the comparison of the results from computer simulations and the AGA analysis presented in Section 3 for the uplink MRC receiver in a Rayleigh fading channel with different numbers of active users. The number of subcarriers and maximum number of users used in the simulation system is 8 . As we can see from Figure 2, the results achieved by the AGA analysis agree well with those of the computer simulations. Similar results can be obtained from Figure 3, which demonstrates the performance comparison of the same approach for the EGC receiver of the uplink in a Rayleigh fading channel. From Figures 2 and 3, it is not difficult to see that the AGA analysis gives nearly the same results as the computer simulations. There exists a marginal difference in the multiple user cases when MAI becomes the dominant factor affecting system performance. This is due to the inadequate assumption of a Gaussian MAI

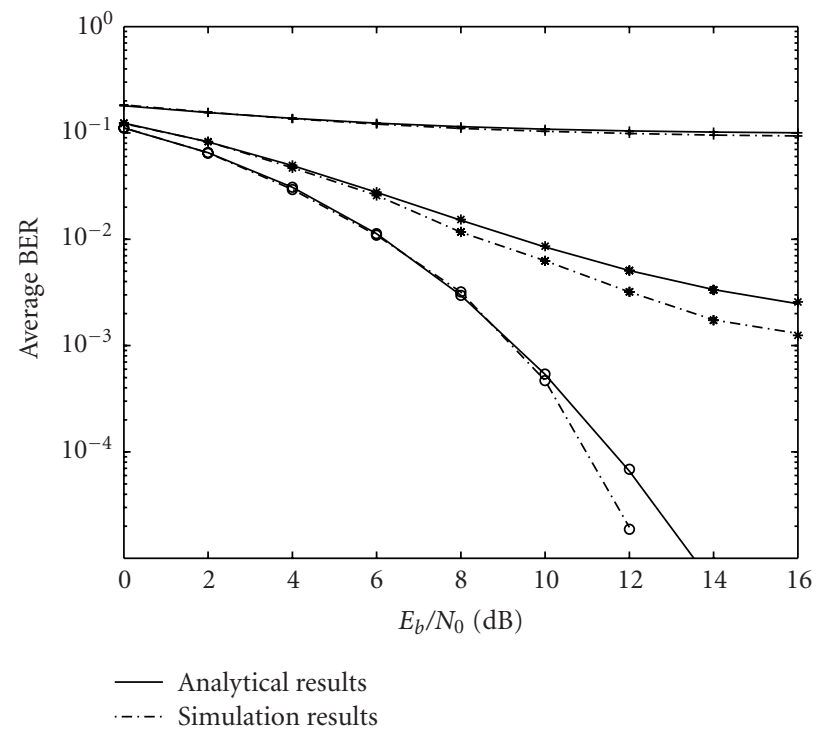

FIGURE 3: BER as a function of $E_{b} / N_{0}(\mathrm{~dB})$ for the EGC receivers of the uplink with different numbers of users in a fading channel. The methods used are AGA analysis and computer simulations $(N=8$; $\circ$ : single user; $*: 2$ active users; $+: 8$ active users).

model when the number of active users is small $(K<10)$. In [8], we have compared the analytical results for EGC receivers using the proposed AGA technique and the method proposed in [8]. It was shown that the two analytical methods give quite the same analysis results from which the accuracy of the presented analysis method was further demonstrated. However, the method presented in $[8]$ requires that the fading channels on all subcarriers have the same fading parameters.

The approach presented in this paper can also be employed to obtain the BER for the receivers in the downlink of an MC-CDMA system. Of course the formulas presented in Section 3 must be changed to correspond to the synchronous downlink case. The performance comparison between the analytical and simulation results of both an MRC receiver and EGC receiver in the downlink are shown in Figures 4 and 5 , respectively. The number of subcarriers is fixed to 8 and the number of active users is varied. It is clearly seen from these two figures that the approach is also accurate in the downlink. MRC is not practical for the downlink as the loss of orthogonality of the WH codes is emphasized in the receiver when applying it. In the downlink, EGC outperforms MRC in most cases, especially at high SNR. This means that the loss of orthogonality for EGC, which is caused by channel fading, is less than that of MRC.

In order to further verify the accuracy of the proposed AGA method, the comparison between analysis and simulation results for Nakagami- $m$ fading channels with $m=2$ is shown in Figure 6 (MRC receivers) and Figure 7 (EGC receiver). The considered system is uplink MC-CDMA with 8 subcarriers and different numbers of active users. From these two figures it should be noted that the AGA method gives more accurate results with $m=2$ than in Rayleigh fading channels. 


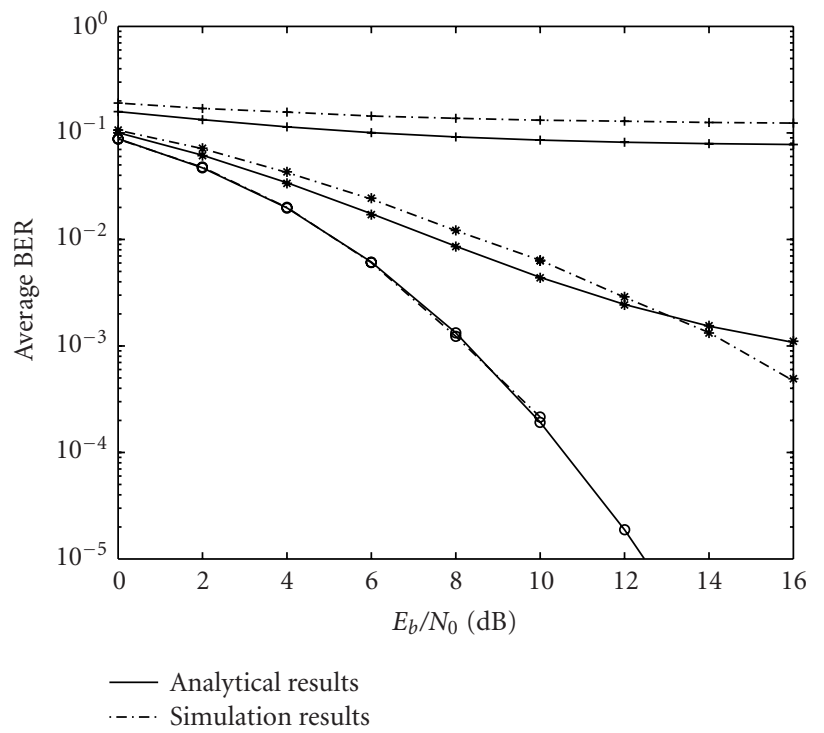

FIgURE 4: BER as a function of $E_{b} / N_{0}(\mathrm{~dB})$ for the MRC receivers of the downlink with different numbers of users in a fading channel. The methods used are AGA analysis and computer simulations $(N=8 ; \circ$ : single user; $*: 2$ active users; $+: 8$ active users $)$.

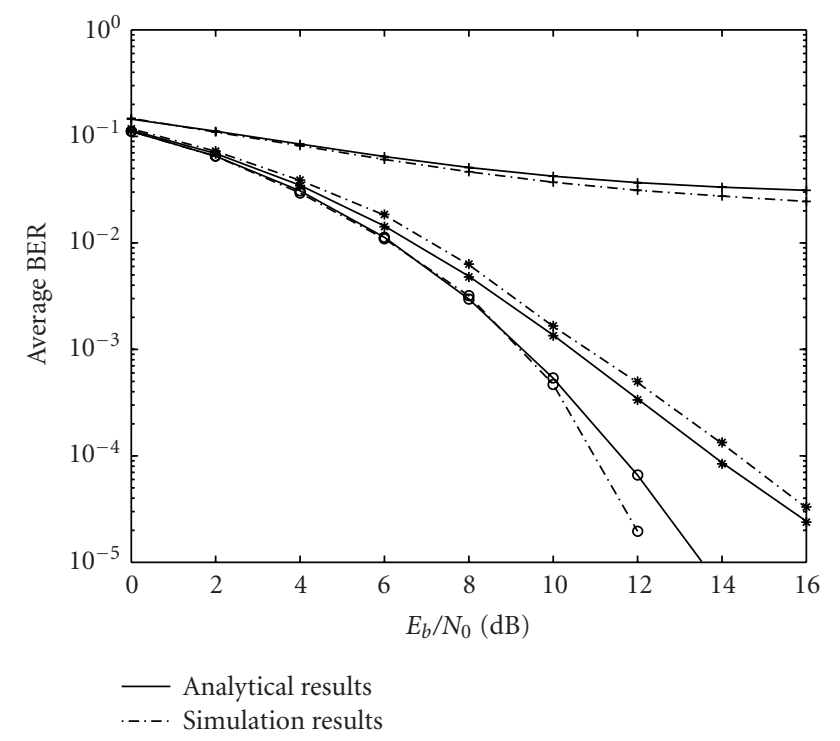

FIgURE 5: BER as a function of $E_{b} / N_{0}(\mathrm{~dB})$ for the EGC receivers of the downlink with different numbers of users in a fading channel. The methods used are AGA analysis and computer simulations $(N=8 ; \circ$ : single user; $*: 2$ active users; $+: 8$ active users $)$.

Figures 8 and 9 illustrate the effects of channel fading parameter $m$ on the performance of MRC and EGC receivers. Both figures show the analytical results for MRC and EGC receivers in the uplink with different numbers of users. The $E_{b} / N_{0}$ is fixed to $0 \mathrm{~dB}$ and the number of subcarriers $N$ is 8 . As expected, the system performance improves as

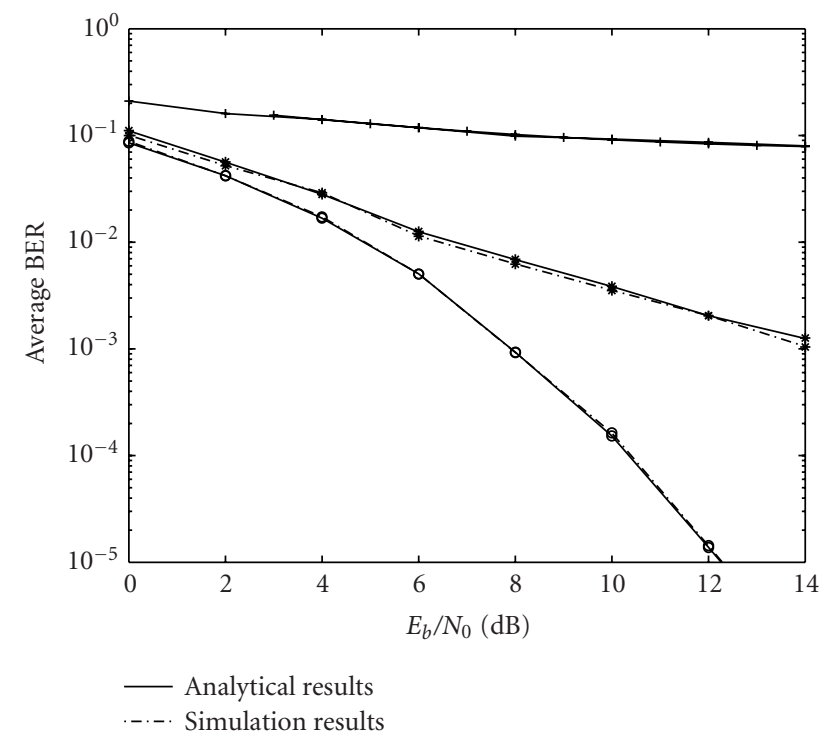

FIGURE 6: BER as a function of $E_{b} / N_{0}(\mathrm{~dB})$ for the MRC receivers of the uplink with different numbers of users in a fading channel. The methods used are AGA analysis and computer simulations $(N=8$; fading parameter $m=2$; $\circ$ : single user; $*: 2$ active users; $+: 8$ active users).

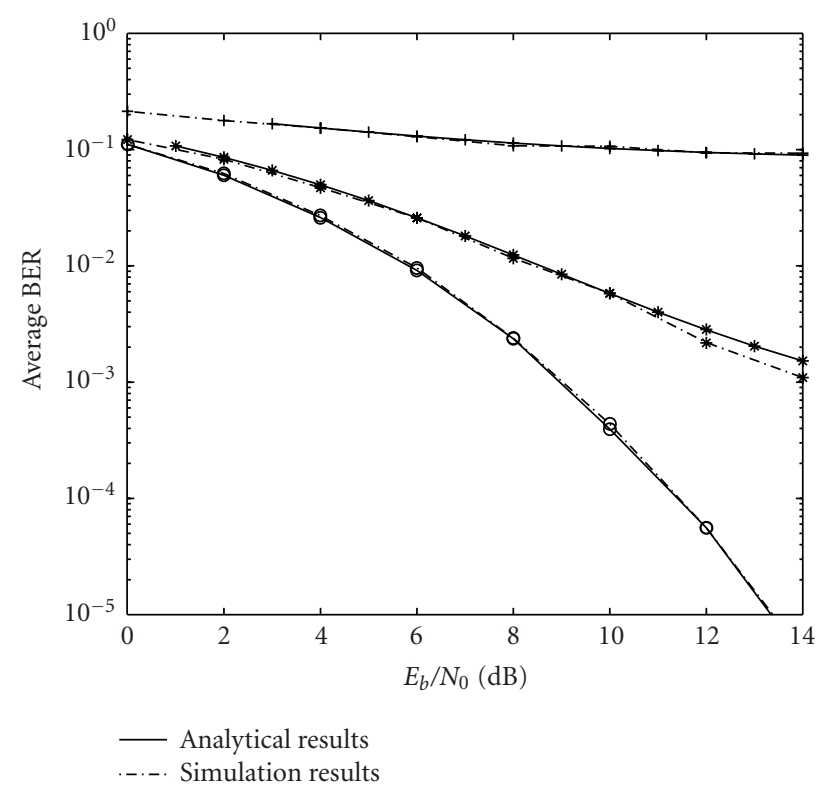

FIGURE 7: BER as a function of $E_{b} / N_{0}(\mathrm{~dB})$ for the EGC receivers of the uplink with different numbers of users in a fading channel. The methods used are AGA analysis and computer simulations $(N=8$; fading parameter $m=2$; $\circ$ : single user; $*: 2$ active users; $+: 8$ active users).

the amount of fading decreases, more specifically, as $m$ increases, the performance of both EGC and MRC receivers becomes better. The performance of the receivers with the nonfading channel can be obtained until $m$ approaches 10. By 


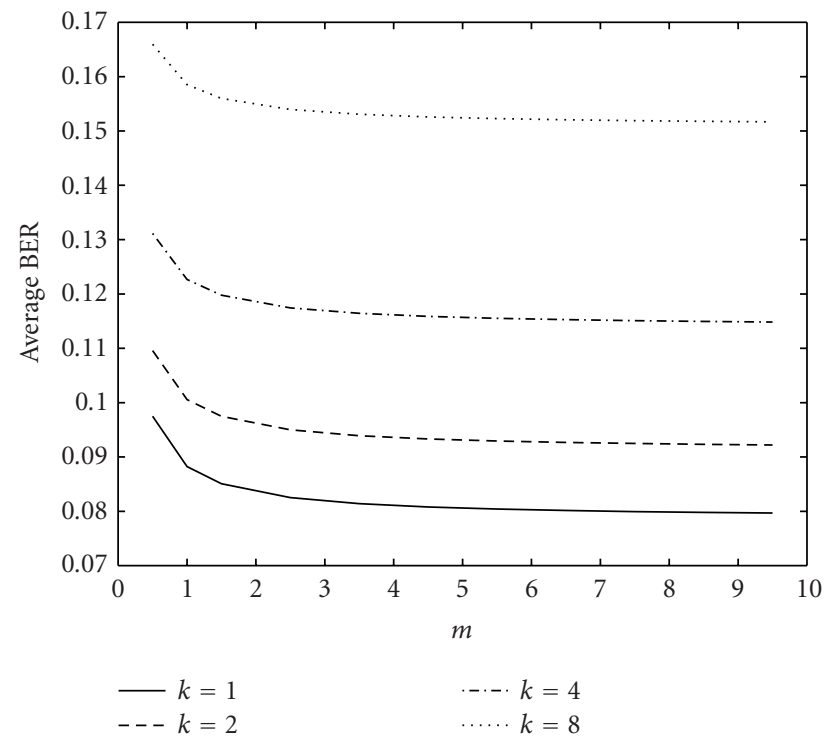

FIGURE 8: BER as a function of the fading parameter $m$ for the MRC receiver of the uplink with different numbers of active users $(N=8$, $\left.E_{b} / N_{0}=0 \mathrm{~dB}\right)$.

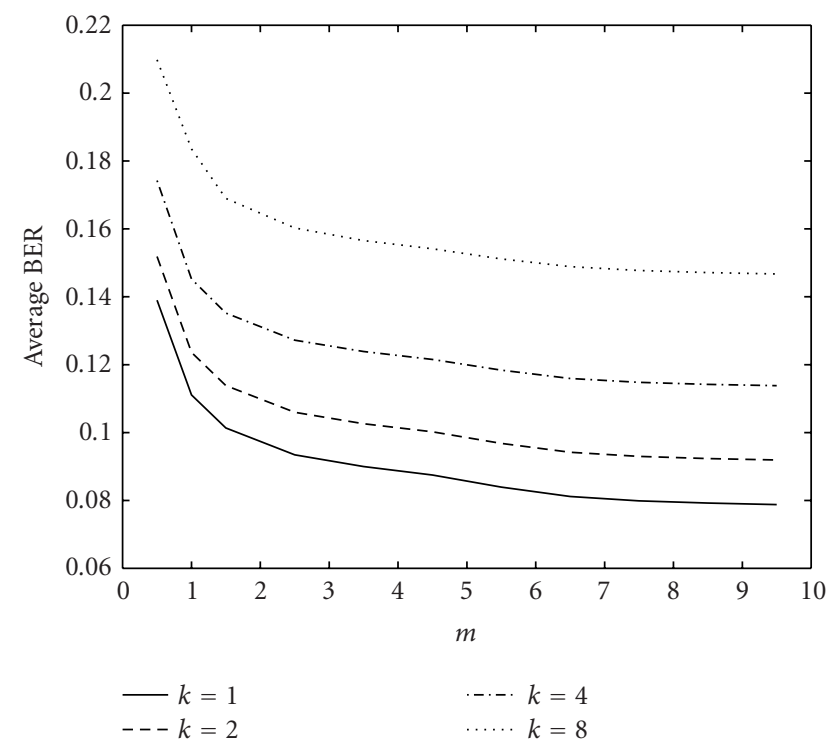

FIGURE 9: BER as a function of the fading parameter $m$ for the EGC receiver of the uplink with different numbers of active users $(N=8$, $\left.E_{b} / N_{0}=0 \mathrm{~dB}\right)$.

comparing the $\mathrm{MRC}$ receiver and the EGC receiver, it should be noted that the performance curves of the EGC receiver change more than that of the MRC receiver and this suggests that the EGC receiver is more sensitive to the variation of fading parameter $m$ than the MRC receiver.

Using the expression for the BER obtained for the uplink transmission in a Nakagami- $m$ fading channel, the average BER versus the number of active users both for EGC

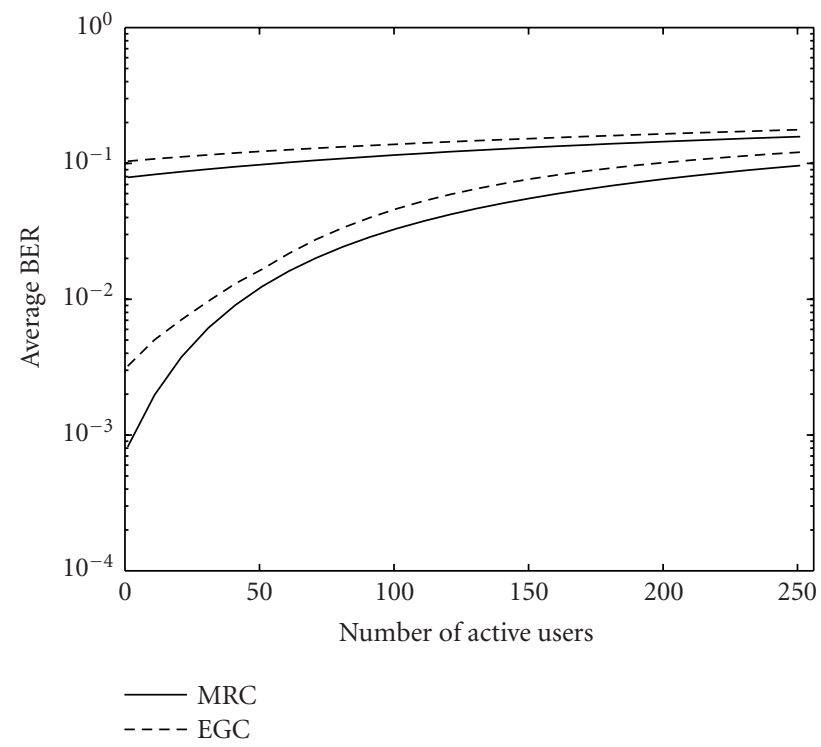

Figure 10: BER as a function of the number of active users for both the EGC and MRC receivers of the uplink in a Rayleigh fading channel $\left(N=256, E_{b} / N_{0}=0 \mathrm{~dB}\right.$ and $\left.7 \mathrm{~dB}\right)$.

and MRC receivers with 256 subcarriers in a Rayleigh fading channel $(m=1)$ is shown in Figure 10 . The $E_{b} / N_{0}$ is fixed at 0 and $7 \mathrm{~dB}$. The significant impacts of MAI can be observed. It can also be noted that at $E_{b} / N_{0}=7 \mathrm{~dB}$, the fully loaded system works well if efficient channel coding is employed.

\section{CONCLUSIONS}

The BER analysis for MC-CDMA receivers with multiple active users in frequency-selective Nakagami- $m$ fading channels was presented in this paper. The analysis was applied to evaluate the performance of both EGC and MRC receivers in the uplink and downlink. The AGA approach utilizes an alternative expression for the $Q$-function, combining this with the characteristic function of Nakagami- $m$ r.v.'s, thereby eliminating the need for deriving the distribution of the sum of Nakagami-m signals for the EGC receiver and, hence, avoiding all approximations required therein. The approach used in this paper has the advantage of simplicity in expression and computational efficiency. Both theoretical analysis and computer simulations were used to evaluate the BER performance of the receivers in Rayleigh fading channels. It was of importance to observe that the computer simulations demonstrated the accuracy of the analysis method based on AGA. Therefore, the method presented here provides us with a powerful practical tool to evaluate the BER performance of MC-CDMA systems, especially when the number of subcarriers and users is too large to obtain simulation results. In addition, it was also seen that the influence of MAI on the system performance is significant and that the BER saturates at high SNR for both EGC and MRC receivers in the uplink and downlink when the system is heavily loaded. 


\section{APPENDIX}

\section{THE CHARACTERISTIC FUNCTION OF A RAYLEIGH RANDOM VARIABLE}

To obtain the performance of the receivers using EGC in a Rayleigh fading, we can let $m_{0, n}=1$ in Section 3 . Alternatively, it can be obtained by directly using the characteristic function of Rayleigh r.v.'s.

The characteristic function of Rayleigh random variables can be expressed by virtue of the sine and cosine transforms,

$$
\begin{aligned}
\psi_{\beta_{0, n}}(j v)= & \int_{0}^{\infty} \frac{2 \beta_{0, n}}{\Omega_{0, n}} \exp \left(\frac{-\beta_{0, n}^{2}}{\Omega_{0, n}}\right) \cos \left(\beta_{0, n} v\right) d \beta_{0, n} \\
& +j \int_{0}^{\infty} \frac{2 \beta_{0, n}}{\Omega_{0, n}} \exp \left(\frac{-\beta_{0, n}^{2}}{\Omega_{0, n}}\right) \sin \left(\beta_{0, n} v\right) d \beta_{0, n} \\
= & { }_{1} F_{1}\left(1 ; \frac{1}{2} ;-\frac{\Omega_{0, n} v^{2}}{4}\right) \\
& +j \sqrt{\frac{\pi \Omega_{0, n}}{4}} v \exp \left(-\frac{\Omega_{0, n} v^{2}}{4}\right) .
\end{aligned}
$$

The following formulas from [23] have been used

$$
\begin{aligned}
& \int_{0}^{\infty} x^{v} \exp \left(-\alpha x^{2}\right) \cos (x y) d x \\
& \quad=\frac{1}{2} \alpha^{-(1 / 2)(1+v)} \Gamma\left(\frac{1}{2}+\frac{1}{2} v\right) \times{ }_{1} F_{1}\left(\frac{1}{2}+\frac{1}{2} v ; \frac{1}{2} ;-\frac{y^{2}}{4 \alpha}\right), \\
& \int_{0}^{\infty} x \exp \left(-\alpha x^{2}\right) \sin (x y) d x \\
& =\frac{1}{4} \alpha^{-(1 / 2)(1+v)} \sqrt{\pi} a^{-3 / 2} y \exp \left(-\frac{1}{4} a^{-1} y^{2}\right) .
\end{aligned}
$$

The characteristic function of a Rayleigh random variable can also be written as

$$
\begin{aligned}
\psi_{\beta_{0, n}}(j v)= & \left({ }_{1} F_{1}\left(-\frac{1}{2} ; \frac{1}{2} ; \frac{\Omega_{0, n} v^{2}}{4}\right)+j \sqrt{\frac{\pi \Omega_{0, n}}{4} v}\right) \\
& \times \exp \left(-\frac{\Omega_{0, n} v^{2}}{4}\right),
\end{aligned}
$$

where the property [21]

$$
{ }_{1} F_{1}(a, b, z)=e^{z} \times{ }_{1} F_{1}(b-a, b, z),
$$

was used. Then following the same procedure as in Section 3 and making some changes, the performance of EGC in a Rayleigh fading channel can be obtained.

\section{ACKNOWLEDGMENTS}

The authors would like to acknowledge Dr. Mohammed Abdel-Hafez from United Arab Emirates University for useful discussions when preparing this paper. The reviewers are appreciated for their helpful comments and suggestions. This paper was presented in part at the IEEE International Conference on Communications (ICC '02), New York, April 28May 2, 2002. This research was supported by the Academy of Finland, the Finnish National Technology Agency (TEKES), Nokia, the Finnish Defence Forces, and Elektrobit.

\section{REFERENCES}

[1] K. Fazel and S. Kaiser, Eds., Multi-Carrier Spread-Spectrum \& Related Topics, Kluwer Academic, Boston, Mass, USA, 2002.

[2] S. Hara and R. Prasad, "Overview of multicarrier CDMA," IEEE Communications Magazine, vol. 35, no. 12, pp. 126-133, 1997.

[3] N. Yee, J.-P. Linnartz, and G. Fettweis, "Multicarrier CDMA in indoor wireless radio networks," in Proc. IEEE Personal, Indoor and Mobile Radio Communications (PIMRC '93), pp. 109-113, Yokohama, Japan, September 1993.

[4] X. Gui and T. S. Ng, "Performance of asynchronous orthogonal multicarrier CDMA system in frequency selective fading channel," IEEE Trans. Communications, vol. 47, no. 7, pp. 1084-1091, 1999.

[5] J. Jang and K. B. Lee, "Effects of frequency offset on MC/CDMA system performance," IEEE Communications Letters, vol. 3, no. 7, pp. 196-198, 1999.

[6] C. K. Kim and Y. S. Cho, "Performance of a wireless MCCDMA system with an antenna array in a fading channel: reverse link," IEEE Trans. Communications, vol. 48, no. 8, pp. 1257-1261, 2000.

[7] S. Moon, G. KO, and K. Kim, "Performance analysis of orthogonal multicarrier-CDMA on two-ray multipath fading channels," IEICE Transactions on Communications, vol. E84B, no. 1, pp. 128-133, 2001.

[8] Z. Li and M. Latva-aho, "BER performance evaluation for MC-CDMA systems in Nakagami- $m$ fading," Electronics Letters, vol. 38, no. 24, pp. 1516-1518, 2002.

[9] N. C. Beaulieu, "An infinite series for the computation of the complementary probability distribution function of a sum of independent random variables and its application to the sum of Rayleigh random variables," IEEE Trans. Communications, vol. 38, no. 9, pp. 1463-1474, 1990.

[10] B. Smida, C. L. Despins, and G. Y. Delisle, "MC-CDMA performance evaluation over a multipath fading channel using the characteristic function method," IEEE Trans. Communications, vol. 49, no. 8, pp. 1325-1328, 2001.

[11] M. K. Simon and M.-S. Alouini, "A unified performance analysis of digital communication with dual selective combining diversity over correlated Rayleigh and Nakagami- $m$ fading channels," IEEE Trans. Communications, vol. 47, no. 1, pp. 3343, 1999.

[12] Q. T. Zhang, "Exact analysis of postdetection combining for DPSK and NFSK systems over arbitrarily correlated Nakagami channels," IEEE Trans. Communications, vol. 46, no. 11, pp. 1459-1467, 1998.

[13] M. Nakagami, "The $m$-distribution-A general formula of intensity distribution of rapid fading," in Statistical Methods in Radio Wave Propagation, pp. 3-36, Pergamon Press, Oxford, UK, 1960.

[14] S. Kaiser, Multi-carrier CDMA mobile radio system-analysis and optimization of detection, decoding, and channel estimation, Ph.D. dissertation, University of Munich, Munich, Germany, 1998.

[15] Z. Li and M. Latva-aho, "Performance comparison of frequency domain equalizer for MC-CDMA systems," in Proc. IEEE International Conference on Mobile and Wireless 
Communications Networks (MWCN '01), pp. 85-89, Recife, Brazil, August 2001.

[16] M. K. Simon and M.-S. Alouini, "A unified approach to the performance analysis of digital communication over generalized fading channels," Proceedings of the IEEE, vol. 86, no. 9, pp. 1860-1877, 1998.

[17] A. Papoulis, Probability, Random Variables, and Stochastic Processes, McGraw-Hill, New York, NY, USA, 3rd edition, 1991.

[18] J. G. Proakis, Digital Communications, McGraw-Hill, New York, NY, USA, 3rd edition, 1995.

[19] I. S. Gradshteyn and I. M. Ryzhik, Table of Integrals, Series, and Products, Academic Press, San Diego, Calif, USA, 5th edition, 1994.

[20] M.-S. Alouini and M. K. Simon, "Performance analysis of coherent equal gain combining over Nakagami- $m$ fading channels," IEEE Trans. Vehicular Technology, vol. 50, no. 6, pp. 1449-1463, 2001.

[21] M. Abramowitz and I. A. Stegun, Handbook of Mathematical Functions with Formulas, Graphs, and Mathematical Tables, Dover Publications, New York, NY, USA, 10th edition, 1972.

[22] Z. Kopal, Numerical Analysis, Chapman \& Hall, London, UK, 2nd edition, 1961.

[23] A. Erdelyi, Ed., Tables of Integral Transforms, vol. 1, McGrawHill, New York, NY, USA, 1954.

Zexian Li received the Ph.D. degree from Beijing University of Posts and Telecommunications, Beijing, China in 1999. Before that, he received the B.S. and M.S. degrees from Harbin Institute of Technology, Harbin, China, in 1994 and 1996, respectively. From August 1999 to September 2000, he was a Research Engineer in Huawei Technologies Co. Ltd., Beijing. Since October 2000, he has been working in Centre for

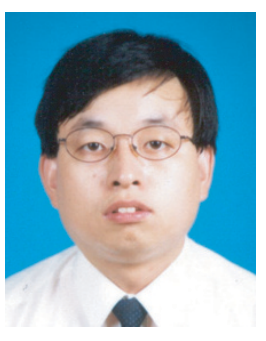
Wireless Communications (CWC) at University of Oulu, Finland. His research interests include future broadband wireless communications, multicarrier communication systems, communication theory, information theory, and advanced signal processing for communications.

Matti Latva-aho received the M.S. (E.E.), Lic.Tech., and Dr. Tech degrees from the University of Oulu, Finland in 1992, 1996, and 1998, respectively. From 1992 to 1993 , he was a Research Engineer at Nokia Mobile Phones, Oulu, Finland. During the years 1994-1998 he was a Research Scientist at Telecommunication Laboratory and Centre for Wireless Communications at the University of Oulu. Prof. Latva-aho has been

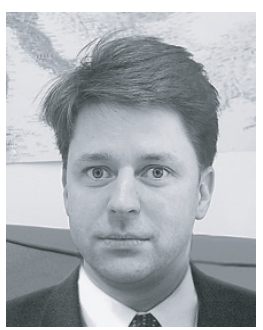
Director of Centre for Wireless Communications at the University of Oulu during 1998-2003. Since 2000 he has been Professor of digital transmission techniques at Telecommunications Laboratory. His research interests include future broadband wireless communication systems and related transceiver algorithms. Prof. Latvaaho has published more than 70 conference or journal papers in the field of CDMA communications. 